\title{
Effects of Ingested Fructooligosaccharides on Stool Frequency in Healthy Female Volunteers: A Placebo-Controlled Study
}

\author{
Shigeru Tominaga, ${ }^{1}$ Masao Hirayama, ${ }^{1}$ Takashi Adachl, ${ }^{1}$ Takahisa Tokunaga ${ }^{2}$ and Hisakazu Iino ${ }^{3}$ \\ 'Bioscience Laboratories, Meiji Seika Kaisha, Ltd., 5-3-1 Chiyoda, Sakado, Saitama 350-0289, Japan \\ ${ }^{2}$ New Materials Division, Meiji Seika Kaisha, Ltd., 580 Horikawa-cho, Saiwai-ku, Kawasaki, Kanagawa 210-0913, Japan \\ ${ }^{3}$ Showa Women's University, 1-7 Taishido, Setagaya-ku, Tokyo 154-0004, Japan
}

Received December 11, 1998; Accepted for publication, March 2, 1999

The effects of ingested fructooligosaccharides (FOS) ( $3 \mathrm{~g} /$ day) and placebo on stool frequency and stool condition were investigated in healthy female volunteers. According to stratification analysis, the stool frequency during the period of FOS intake was: 1) higher than that during the period of non-intake comparing subjects with an inherent (non-intake period) stool frequency of $\leq 3$ times/week; 2) higher than that during the period of placebo intake comparing those subjects with an inherent frequency of $>3$ to $\leq 5$ times/week; and 3) not higher or lower, with no significant differences observed between the three periods, comparing those subjects with an inherent frequency of $>5$ times/week. Three statistical methods were applied in the analyses, and comparisons revealed that the non-parametric Wilcoxon signed rank-sum test was suitable in this study.

Key words: fructooligosaccharides; stool frequency; volunteer; placebo; human

\section{INTRODUCTION}

In the past decade, nondigestible oligosaccharides have been found to have beneficial effects with respect to intestinal conditions and defecation $(2-7,9,10)$, and several of them have been approved as "Foods for Specified Health Use" to increase stool frequency. For confirmation of their usefulness, various experimental designs and statistical methods have been proposed, but as yet there has been no placebo-controlled evaluation except for one study recently reported by Deguchi et al. concerning the administration of galactooligosaccharides (1). Analysis of the effect through comparison with a placebo group by suitable statistical methods is important in evaluating the physiological and psychological effects of intake of nondigestible oligosaccharides.

In a previous study (9), we found that FOS-intake increased stool frequency significantly $(p<0.05)$ in healthy volunteers. However, the significance was recognized using a paired $t$-test for comparison of a FOSintake group comprised of all subjects given FOS at three dose levels $(1,3$, or $5 \mathrm{~g} /$ day $)$ and a non-intake group. In this report, we describe the effects of the intake of a single daily dose of FOS ( $3 \mathrm{~g} /$ day) as compared to placebo intake in healthy female volunteers. The study was conducted with the following objectives: 1) to evaluate the effects on stool frequency and stool condition including the quantity, hardness, color, and transit time; 2) to compare the findings for three peri- ods (i.e., the FOS-, placebo- and non-intake periods) for each of three classes of subjects stratified according to the inherent (non-intake period) stool frequency; and 3) to test for significant differences using three different statistical methods.

\section{MATERIALS AND METHODS}

Sample preparation. The test materials were administered in tablet form. The composition of the tablets used is shown in Table 1 . The FOS $\left(1^{\mathrm{F}}-(\beta-\right.$ fructofuranosyl) ${ }_{n}$ sucrose, $n=2-4$ ) employed in this study, consisting of 1-kestose $(n=2,30 \%)$, nystose $(n=$ $3,57 \%)$, and $1^{\mathrm{F}}$-( $\beta$-fructofuranosyl)nystose $(n=4$, $14 \%$ ), was a commercial product, Meioligo ${ }^{\circledR} \mathrm{P}$ (Meiji Seika Kaisha, Ltd., Tokyo; content of FOS was greater than $95 \%$ of the dry materials and the moisture content was 5\%). Each type of tablet had no other ingredients that might contribute to the improvement of constipation such as other nondigestible saccharides, dietary fiber, lactose, milk products, or Bifidobacterium, and the sweetness of each type of tablet was adjusted to the same level with Aspartam ${ }^{\circledR}$ (Ajinomoto Co., Tokyo). Each subject was given 5 tablets $(10 \mathrm{~g})$ per day orally, which corresponded to $3 \mathrm{~g}$ of FOS per day during the FOS-intake period.

Subjects. The study was reviewed and approved by the Clinical Studies Committee of Showa Women's University, and signed informed consent was obtained from each subject before participation. Seventy-five female students, 18-21 years old, were recruited, and 
Table 1. Composition [w/w(\%)] of the test tablets.

\begin{tabular}{lrr}
\hline & Placebo & \multicolumn{1}{c}{3 g/day } \\
\hline Meioligo $^{\circledR}$ & 0.000 & 33.000 \\
Sugar $_{\text {Citric acid }}$ & 84.747 & 54.242 \\
Flavors & 5.000 & 5.000 \\
Aspartam $^{\circledR}$ & 7.253 & 4.642 \\
Sugar esters $_{\text {Wax }}$ & 0.000 & 0.116 \\
& 2.000 & 2.000 \\
& 1.000 & 1.000 \\
\hline
\end{tabular}

Table 2. Characteristics of the subjects.

\begin{tabular}{llll}
\hline & $\begin{array}{l}\text { Normal } \\
\text { group } \\
(n=12)\end{array}$ & $\begin{array}{c}\text { Lower } \\
\text { group } \\
(n=9)\end{array}$ & $\begin{array}{c}\text { Lowest } \\
\text { group } \\
(n=13)\end{array}$ \\
\hline Age (year) & $20.0 \pm 1.0^{* 1}$ & $19.8 \pm 0.8$ & $19.8 \pm 1.0$ \\
Body weight (kg) & $51.3 \pm 5.5$ & $48.0 \pm 2.8$ & $48.9 \pm 3.1$ \\
$\begin{array}{c}\text { Stool frequency } \\
\text { (number/week) }\end{array}$ & $7.13 \pm 1.38$ & $4.28 \pm 0.62$ & $2.77 \pm 0.33$ \\
\hline
\end{tabular}

${ }^{*}$ I Mean \pm S.D.

prior to the main study a preliminary screening test, to identify any volunteers showing an irregular frequency of evacuation and to assess the degree of the placebo effect, was performed for each volunteer through a 7 day placebo-intake period followed by a 20 -day period of non-intake and then a 7-day FOS-intake period. After exclusion of the volunteers who displayed irregular stool frequencies, 43 volunteers were selected as subjects for the main study. Excluding those who dropped out, 34 subjects took part in this study and provided full records of their stool frequency and stool condition. Characteristics of the subjects are shown in Table 2:

Experimental design. All subjects were given test tablets ( 5 pieces/day) containing FOS or placebo, and neither the content of the tablets nor the group assignments were known to any of the subjects. Actually, the first period of 14 days was used for the administration of FOS ( $3 \mathrm{~g} /$ day), the following 14 days was the period of non-intake, and the last 14 days was the period of placebo administration. During the test period, each subject participated in this study in a free-living state and recorded each occurrence of evacuation (frequency), stool condition (quantity, hardness, and color) and general health condition, as described below. Regarding the transit time, this was measured on the middle day of each of the three test periods (the FOS-, placeboand non-intake periods) by the following method. Each subject took 50 white colored cellulose pills (about $5 \mathrm{~mm}$ in diameter) coated with zein to measure transit time. Since zein is scarcely digestible, the subjects could easily find these pills in their feces.

Assessment. The assessment was made by questionnaire. Each subject recorded the occurrence of evacuation (frequency), stool condition (quantity, hardness, and color), and general health condition every day, as instructed in the preliminary test. They also recorded the intake of any supplements for constipation such as yogurt or dietary fiber and the intake of any medicines such as antibiotics or other drugs. The items of quantity, hardness, and color of stools were converted to numerical data as follows. Stool quantity was measured as the number of equivalents to the amount of one egg, and the hardness and color of stools were distinguished and rated according to six numerical grades (hardness: 1 (very hard), 2 (hard), 3 (medium), 4 (soft), 5 (muddy), and 6 (watery); color (using the Munsell Color System): 1 (yellowish, 2.5Y 5/8), 2 (light-golden, 7.5YR 5/8), 3 (dark-golden, 5 YR 4/6), 4 (brownish, 5 YR 2/3), 5 (dark-brownish, 7.5YR 3/2), and 6 (blackish, 10YR $2 / 1)$ ). All of the data were collected and reviewed by the investigators responsible for this study. During the main study, nine subjects dropped out and their data were excluded.

Statistical methodology. All of the records were transformed to numerical data prior to formal analysis. In stratifying the data, each subject was assigned to one of three classes on the basis of the stool frequency observed for the period of non-intake in the main study ("normal": > 5 times/week; "lower": $>3$ to $\leq 5$ times/ week; and "lowest": $\leq 3$ times/week). Differences were investigated statistically comparing the findings for the FOS-intake, non-intake, and placebo-intake periods for these three classes of subjects. Stool frequency and the grade of fecal hardness and color were expressed as average values for each group and period. The results were statistically analyzed using the Wilcoxon signed rank-sum test as the first choice, followed by comparison of differences by other statistical methods, a nonparametric signed test, and a parametric paired $t$-test. Table 4 shows the $p$-values for stool frequency for the three statistical tests.

\section{RESULTS}

Average values for stool frequency, stool condition (quantity, hardness, and color), and transit time were summarized for the three test periods (FOS intake, nonintake, and placebo intake) for the three classes of subjects separated according to their inherent (non-intake 
Table 3. Average values for frequency of evacuation, stool condition (quantity, hardness, and color), and transit time in three test periods (FOS, non-intake, and placebo) for three classes of subjects separated according to their inherent (non-intake period) stool frequency.*1

\begin{tabular}{llccccc}
\hline Class $^{* 2}$ & Test-period & Frequency*3 $^{*}$ & Quantity*4 $^{* 4}$ & Hardness*5 $^{* 3}$ & Color $^{* 6}$ & Transit time*7 $^{*}$ \\
\hline Lowest & Non-intake & $2.8 \pm 0.1^{\mathrm{a}}$ & $5.0 \pm 0.5^{\mathrm{a}}$ & $2.7 \pm 0.1^{\mathrm{a}}$ & $3.5 \pm 0.2^{\mathrm{a}}$ & $54.3 \pm 9.4^{\mathrm{a}}$ \\
$\quad \leq 3$ & Placebo & $3.7 \pm 0.4^{\mathrm{b}}$ & $6.1 \pm 0.9^{\mathrm{ab}}$ & $2.7 \pm 0.1^{\mathrm{a}}$ & $3.4 \pm 0.2^{\mathrm{a}}$ & $58.6 \pm 11.0^{\mathrm{a}}$ \\
$(n=13)$ & FOS & $3.7 \pm 0.3^{\mathrm{b}}$ & $6.7 \pm 0.8^{\mathrm{b}}$ & $2.8 \pm 0.1^{\mathrm{a}}$ & $3.3 \pm 0.2^{\mathrm{a}}$ & $52.5 \pm 5.9^{\mathrm{a}}$ \\
\hline Lower & Non-intake & $4.3 \pm 0.2^{\mathrm{ab}}$ & $9.8 \pm 1.1^{\mathrm{ab}}$ & $3.1 \pm 0.2^{\mathrm{a}}$ & $3.3 \pm 0.2^{\mathrm{a}}$ & $49.9 \pm 11.2^{\mathrm{a}}$ \\
$>3, \leq 5$ & Placebo & $4.3 \pm 0.5^{\mathrm{a}}$ & $10.0 \pm 1.5^{\mathrm{a}}$ & $2.8 \pm 0.2^{\mathrm{a}}$ & $3.4 \pm 0.2^{\mathrm{a}}$ & $45.6 \pm 14.4^{\mathrm{a}}$ \\
$(n=9)$ & FOS & $5.3 \pm 0.6^{\mathrm{b}}$ & $11.3 \pm 1.4^{\mathrm{b}}$ & $3.0 \pm 0.2^{\mathrm{a}}$ & $3.1 \pm 0.2^{\mathrm{a}}$ & $30.4 \pm 5.6^{\mathrm{a}}$ \\
\hline Normal & Non-intake & $7.1 \pm 0.4^{\mathrm{a}}$ & $13.9 \pm 1.4^{\mathrm{a}}$ & $3.2 \pm 0.1^{\mathrm{a}}$ & $3.0 \pm 0.2^{\mathrm{a}}$ & $40.7 \pm 9.4^{\mathrm{a}}$ \\
$>5$ & Placebo & $6.5 \pm 0.6^{\mathrm{a}}$ & $13.1 \pm 1.4^{\mathrm{a}}$ & $3.0 \pm 0.2^{\mathrm{a}}$ & $3.0 \pm 0.2^{\mathrm{a}}$ & $30.9 \pm 2.8^{\mathrm{a}}$ \\
$(n=12)$ & FOS & $6.3 \pm 0.5^{\mathrm{a}}$ & $12.7 \pm 1.6^{\mathrm{a}}$ & $2.9 \pm 0.1^{\mathrm{a}}$ & $3.0 \pm 0.2^{\mathrm{a}}$ & $36.6 \pm 10.3^{\mathrm{a}}$ \\
\hline
\end{tabular}

*1 Data are expressed in the form of 'mean \pm S.E.'

Values with different superscripts are significantly different $(p<0.05)$ as determined by the Wilcoxon signed rank-sum test.

Class was stratified according to stool frequency in the non-intake period.

The number of samples are 9 (class ' $\leq 3$ '), 5 (class ' $>3, \leq 5$ ') and 10 (class ' $>5$ ') for transit time.

${ }^{2}$ Stool frequency in the non-intake period: times per week.

*3 Times per week.

${ }^{* 4}$ Units per week, 1 unit is the volume of an egg.

$* 51$, very hard; 2 , hard; 3 , medium; 4 , soft; 5 , muddy; 6 , watery.

*6 1, yellowish; 2, light-golden; 3, dark-golden; 4, brownish; 5, dark-brownish; 6, blackish.

${ }^{* 7}$ Hours.

Table 4. $P$-value for each statistical treatment used for comparisons of stool frequency.*1

\begin{tabular}{|c|c|c|c|c|c|c|c|c|c|}
\hline \multirow[b]{2}{*}{ Comparison } & \multirow[b]{2}{*}{ Class } & \multicolumn{3}{|c|}{$P$-value of statistical methods } & \multirow{2}{*}{$\begin{array}{l}\text { Number } \\
\text { of } \\
\text { subject }\end{array}$} & \multicolumn{3}{|c|}{ Average $* 5$} & \multirow{2}{*}{$\begin{array}{c}\text { Ratio of } \\
\text { variation to } \\
\text { the whole }{ }^{* 6} \\
(\%)\end{array}$} \\
\hline & & Wilcoxon*2 & Signed*3 & Paired $t^{* 4}$ & & FOS & Placebo & $\begin{array}{l}\text { Non- } \\
\text { intake }\end{array}$ & \\
\hline FOS & Lowest & 0.836 & 0.754 & - & 13 & 3.69 & 3.65 & & 4 \\
\hline vs. & Lower & $0.007 *$ & $0.004 *$ & - & 9 & 5.33 & 4.28 & & 100 \\
\hline Placebo & Normal & 0.591 & 0.227 & - & 12 & 6.25 & 6.50 & & 一 \\
\hline Placebo & Lowest & $0.019 *$ & 0.065 & $0.020 *$ & 13 & & 3.65 & 2.77 & 96 \\
\hline vs. & Lower & 0.932 & 1.000 & 1.000 & 9 & & 4.28 & 4.28 & 0 \\
\hline Non-intake & Normal & 0.293 & 0.754 & - & 12 & & 6.50 & 7.13 & - \\
\hline FOS & Lowest & $0.012 *$ & 0.065 & $0.008 *$ & 13 & 3.69 & & 2.77 & \\
\hline vs. & Lower & 0.107 & 0.125 & - & 9 & 5.33 & & 4.28 & \\
\hline Non-intake & Normal & 0.065 & 0.060 & - & 12 & 6.25 & & 7.13 & \\
\hline
\end{tabular}

${ }^{* 1}$ Values with an asterisk are significantly different $(p<0.05)$. Class was stratified according to stool frequency in the nonintake period.

*2 Wilcoxon signed rank-sum test, non-parametric.

*3 Signed test, non-parametric.

*4 Paired $t$-test, parametric. The paired $t$-test was employed when the normality was assured as determined by calculation of the skewness and kurrosis, and the symbol (-) indicates that the normality of the distribution of the population is not assured.

*5 The stool frequency: times per week.

*6 In the "normal" class, the ratio of variation wasn't calculated, because in the FOS-intake period, the frequency was less than that in the non-intake period. 
period) stool frequency (Table 3). The averages of stool frequency during the non-intake, placebo-intake and FOS-intake periods for the subjects in the "lowest" frequency class ( $\leq 3$ times/week) were $2.8 \pm 0.1,3.7 \pm$ 0.4 , and $3.7 \pm 0.3$; those for the subjects in the "lower" frequency class ( $>3$ to $\leq 5$ times/week) were $4.3 \pm 0.2$, $4.3 \pm 0.5$ and $5.3 \pm 0.6$; and those for subjects in the "normal" class $>5$ times/week were $7.1 \pm 0.4,6.5 \pm$ 0.6 and $6.3 \pm 0.5$ (times/week). The Wilcoxon signed rank-sum test revealed the following. For the subjects in the "lowest" frequency class, there was a significant difference in stool frequency comparing the FOS-intake or placebo-intake periods and the period of nonintake, and for subjects in the "lower" frequency class, the stool frequency in the FOS-intake period was significantly higher than in the placebo-intake period. For subjects in the "normal" class, there was no significant difference among the three test periods.

Regarding stool condition, only the quantity was found to change while the two other factors, hardness and color, showed no clear change. The change in stool quantity in this study seemed to be consistent with the change in stool frequency. For subjects in the "lower" frequency class, the average value for the FOS-intake period was $11.3 \pm 1.4$ units/week, which was significantly higher than the value for the placebo-intake period. Moreover, for both the "lowest" and the "normal" class, a statistical difference in quantity among the three test periods was completely consistent with the findings concerning the stool frequency.

The transit time for subjects of each class was found to be within the range of $30.4 \pm 5.6$ and $58.6 \pm 11.0$. No statistical difference in transit time among the three test periods was observed for subjects of any class.

Statistical analysis of stool frequency was performed by three different methods, the Wilcoxon signed ranksum test (non-parametric), signed test (non-parametric), and paired $t$-test (parametric). The $p$-values are summarized in Table 4, showing that statistical difference was recognized most frequently by the Wilcoxon signed rank-sum test.

\section{DISCUSSION}

According to the experimental design employed, the test by questionnaire was continued for 6 weeks. Therefore, some features were introduced in an effort to avoid irregular and strange answers. First, the subject's diet and lifestyle couldn't be controlled for such a long period, so we adopted the preliminary test for selection of appropriate subjects, for whom it appeared that the stool frequency was not irregular. Second, in an effort to reduce the subject's concern about evacuation, the questionnaire consisted of not only the essential items concerning stool frequency and condition but also dummy questions about beauty and physical condition.

Assignment of the subjects to three classes on the basis of stool frequency in the period of non-intake afforded interesting results upon comparison of the data for the FOS-, placebo-, and non-intake periods by the Wilcoxon signed rank-sum test (Table 3). For subjects in the "lowest" frequency class ( $\leq 3$ times/week or less), the stool frequency in the FOS-intake period and that in the placebo-intake period both showed a significant difference compared to that in the period of non-intake while there was no significant difference between the FOS- and placebo-intake periods. For subjects in the "lower" class ( $>3$ to $\leq 5$ times/week), the stool frequency during the period of FOS intake increased significantly compared to that in the placebo-intake period. For subjects in the "normal" class (> 5 times/ week), there was no significant difference among the three periods.

Interpretation of the results of statistical analysis comparing the three classes of subjects suggests the following tendencies: 1) for subjects in the "lowest" class, the stool frequency increased even as a result of placebo intake; 2) for subjects in the "lower" class, a difference between FOS- and placebo-intake was recognized; and 3) for subjects in the "normal" class, the inherent (non-intake period) frequency wasn't affected even by nondigestible oligosaccharide intake. These tendencies are consistent with the results of a recent study comparing galactooligosaccharide and placebo intake, indicating that a significant difference was recognized in only the low stool frequency class (4 times/ week or less) (1). Regarding the items of stool condition, stool quantity for subjects in the "lower" and "lowest" classes showed significant differences, which were very similar in relation to the stool frequencies.

In the analysis of stool frequency described above, we confirmed the following. First, FOS intake in the preceding period had no substantial effect on the stool frequency in the non-intake period, since the stool frequency in the first week of the non-intake period was almost the same as that in the second week of the nonintake period. It seems likely that any trailing effect of FOS intake would have led to a higher frequency in the first week of the non-intake period. Second, the results cannot be explained by the regression to the mean because the mean value for the FOS-intake period for subjects in the "normal" class was smaller than that for non-intake, but the values for subjects in the other two 
Table 5. Previous trials concerning improvement of constipation through intake of oligosaccharides.

\begin{tabular}{llllc}
\hline \multicolumn{1}{c}{ Study } & Object*1 & Control & Statistical result & \multicolumn{1}{c}{ Statistical methods } \\
\hline T. Tokunaga et al. (1993)(9) & FOS & non-intake & effective & paired $t$ \\
K. Kondo et al. (1995)(6) & SOS & non-intake & effective & paired $t$ \\
H. Iino et al. (1995)(3) & LS & non-intake & effective*2 & Wilcoxon signed rank-sum \\
Y. Deguchi et al. (1997)(1) & GOS & placebo & ineffective & paired $t$ \\
T. Hara et al. (1997)(2) & SOS & non-intake & effective & paired $t$ \\
M. Kumemura et al. (1992)(7) & LS & non-intake & ineffective & Tukey \\
\hline
\end{tabular}

${ }^{* 1}$ LS, lactosucrose; GOS, galactooligosaccharides; SOS, soybean oligosaccharides.

${ }^{* 2}$ For the subjects whose stool frequency was 4 times/week or less.

classes on the contrary became larger to the same degree. Third, there was no need to consider the multiplicity because both the non-intake and placebo-intake periods were treated as controls.

The major statistical method used for evaluating the effects of oligosaccharide intake on stool frequency in studies previously reported was the parametric paired $t$-test. Typical studies are summarized in Table 5. However, in operation of the statistical analysis, it is important to adopt a method conforming to the properties of the statistics (8). The data of the present study has the following properties: 1) the stool frequency, stool quantity, and transit time are measured on an interval scale which is discrete; 2) hardness and color are ordinal scale values; and 3) the normality of the samples is not assured. The paired test should be used only when the normality of the distribution of the population is assured. In previous reports, shown in Table 5, the confirmation of normality was not described and seemed to be obscure. In order to compare the conformability of the statistical method to this case, analyses were performed by three representative methods, the Wilcoxon signed rank-sum test (non-parametric), signed test (nonparametric) and paired test (parametric), and resultant $p$-values were compared. As shown in Table 4, the Wilcoxon signed rank-sum test detected statistical difference more frequently than the other two methods, and in the case of the paired test, some $p$-values couldn't be obtained because of a lack of normality. These results suggested that the Wilcoxon test was the most suitable for recognizing the variation in the present study.

\section{REFERENCES}

(1) Deguchi Y, Matsumoto K, Ito A, Watanuki M. 1997. Effects of $\beta-1,4$ galactooligosaccharides administration on defecation of healthy volunteers with constipation tendency. Jpn J Nutr 55: 13-22.

(2) Hara T, Ikeda N, Hatsumi K, Watabe J, lino H, Mitsuoka T. 1997. Effect of small amount of ingestion of soybean oligosaccharides on bowel habits and fecal flora of volunteers. Jpn J Nutr 55: 79-84.

(3) Iino H, Tanaka T, Fujita T, Mikuni K, Hara K. 1995. Effect of oral intake of lactosucrose on defecation in healthy female volunteers. Jpn J Med Pharm Sci 33: 855-862.

(4) Iizuka M, Kito Y, Yamada M. 1987. About the improvement of fecal property of dialytic treating patients. Jpn J Clin Dialysis 3 (10): 123-128.

(5) Kitaoka K, Saito K, Tanaka T, Ogata Y, Hiki R, Fujita T, Mikuni K, Hara K. 1995. Effect of $4^{\mathrm{G}}$ - $\beta$-D-galactosylsucrose (lactosucrose) on improvement of evacuation in comparatively constipated female. J New Remedies \& Clinics 44: 780-788.

(6) Kondo K, Anezaki I, Takeshima H. 1995. Effect of a soy oligosaccharide drink on controlling intestinal function, constipation and defecation. Jpn J Med Pharm Sci 34: 333337.

(7) Kumemura M, Hashimoto F, Fujii C, Matsuo K, Kimura $\mathrm{H}$, Miyazoe R, Okamatsu H, Inokuchi T, Ito H, Oizumi K, Oku T. 1992. Effects of administration of $4^{\mathrm{G}}-\beta$-D-galactosylsucrose on fecal microflora, putrefactive products, shortchain fatty acids, weight, moisture and $\mathrm{pH}$, and subjective sensation of defecation in the elderly with constipation. J Clin Biochem Nutr 13: 199-210.

(8) Tango T. 1986. Statistics for Clinical Examination, Asakurashoten, Tokyo, p. 31-33.

(9) Tokunaga T, Nakada Y, Tashiro Y, Hirayama M, Hidaka H. 1993. Effects of fructooligosaccharides intake on the intestinal microflora and defecation in healthy volunteers. Bifidus 6: 143-150.

(10) Wada K, Watabe J, Mizutani J, Suzuki H, Kiryu N, Hayakawa K, Yamaguchi C. 1991. Effects of soybean oligosaccharides intake on fecal microflora, enzyme activity, ammonia and frequency of evacuation in elderly persons. Bifidus 4: 135-140. 